\title{
S100A1: A Multifaceted Therapeutic Target in Cardiovascular Disease
}

\author{
David Rohde • Julia Ritterhoff • Mirko Voelkers • \\ Hugo A. Katus • Thomas G. Parker • Patrick Most
}

Received: 5 May 2010 / Accepted: 2 July 2010 /Published online: 20 July 2010

(C) The Author(s) 2010. This article is published with open access at Springerlink.com

\begin{abstract}
Cardiovascular disease is the leading cause of death worldwide, showing a dramatically growing prevalence. It is still associated with a poor clinical prognosis, indicating insufficient long-term treatment success of currently available therapeutic strategies. Investigations of the pathomechanisms underlying cardiovascular disorders uncovered the $\mathrm{Ca}^{2+}$ binding protein $\mathrm{S} 100 \mathrm{~A} 1$ as a critical regulator of both cardiac performance and vascular biology. In cardiomyocytes, S100A1 was found to interact with both the sarcoplasmic reticulum ATPase (SERCA2a) and the ryanodine receptor 2 (RyR2), resulting in substantially improved $\mathrm{Ca}^{2+}$ handling and contractile performance. Additionally, S100A1 has been described to target the cardiac sarcomere and mitochondria, leading to reduced
\end{abstract}

D. Rohde $\cdot$ J. Ritterhoff $\cdot$ M. Voelkers $\cdot$ H. A. Katus $\cdot$ P. Most Laboratory for Molecular and Translational Cardiology,

Division of Cardiology, Department of Internal Medicine III, University of Heidelberg,

INF 350,

69120 Heidelberg, Germany

M. Voelkers

San Diego Heart Institute, San Diego State University,

San Diego, CA 92182, USA

T. G. Parker

Keenan Research Centre, Li Ka Shing Knowledge Institute,

Division of Cardiology, Department of Medicine,

St. Michael's Hospital, University of Toronto,

30 Bond St.,

Toronto, ON M5B 1W8, Canada

P. Most $(\square)$

Laboratory for Cardiac Stem Cell and Gene Therapy,

Center for Translational Medicine, Department of Medicine,

Thomas Jefferson University,

Philadelphia, PA 19107, USA

e-mail: patrick.most@jefferson.edu pre-contractile passive tension as well as enhanced oxidative energy generation. In endothelial cells, molecular analyses revealed a stimulatory effect of S100A1 on endothelial NO production by increasing endothelial nitric oxide synthase activity. Emphasizing the pathophysiological relevance of S100A1, myocardial infarction in S100A1 knockout mice resulted in accelerated transition towards heart failure and excessive mortality in comparison with wild-type controls. Mice lacking S100A1 furthermore displayed significantly elevated blood pressure values with abrogated responsiveness to bradykinin. On the other hand, numerous studies in small and large animal heart failure models showed that S100A1 overexpression results in reversed maladaptive myocardial remodeling, long-term rescue of contractile performance, and superior survival in response to myocardial infarction, indicating the potential of S100A1-based therapeutic interventions. In summary, elaborate basic and translational research established S100A1 as a multifaceted therapeutic target in cardiovascular disease, providing a promising novel therapeutic strategy to future cardiologists.

Keywords S100A1 · Gene Therapy · Heart Failure · Endothelial Dysfunction · Calcium Cycling

\section{Introduction}

Cardiovascular disease is the leading cause of mortality worldwide, accounting for approximately $30 \%$ of all deaths per year [1, 2]. In 2006, 81,100,000 people suffered from one or more forms of cardiovascular disease in the USA alone [3]. Particularly frightening is evidence for a dramatically growing prevalence of cardiovascular disease, predicting a doubling of lethal outcomes by 2030 [1]. Aside 
from its epidemiological significance, cardiovascular disease represents an increasing socio-economic burden for health care systems all over the world [2].

Although therapeutical options have considerably expanded over the last decade, cardiovascular illnesses, especially heart failure and atherosclerosis, are still associated with a bad clinical prognosis and very little hope for the individual patient [4-6]. Therefore, the decoding of the underlying molecular pathomechanisms with an identification of novel therapeutic targets appears to be urgently necessary.

In this review, we discuss the molecular interactions and regulatory function of the small $\mathrm{Ca}^{2+}$ binding protein S100A1 in cardiomyocytes and endothelial cells with a special focus on its pathophysiological relevance. Finally, we aim to highlight the potential of an S100A1-targeted therapy in cardiovascular disease.

\section{The S100 Protein Family}

S100 proteins represent the largest subgroup within the $\mathrm{Ca}^{2+}$ binding EF-hand protein superfamily [7, 8]. They were first described by Moore et al. in 1965 and represent small acidic proteins with a molecular weight of 9 to $13 \mathrm{kDa}$ that are exclusively found in vertebrates. Due to their unique solubility in a solution of $100 \%$ saturated ammonium sulfate, they were termed "S100" [9]. To date, 25 individual S100 genes have been identified in humans, and most of them, including S100A1, are tightly clustered on chromosome $1 \mathrm{q} 21$ [8]. According to the current nomenclature, S100 genes located in this region are designated "S100A" followed by consecutive Arabic numbers (S100A1, S100A2, S100A3, etc.), whereas genes on other chromosomes like S100B or S100P are given other characters as stem symbol [10].

A common and exceptional characteristic of all S100 proteins is the presence of two $\mathrm{Ca}^{2+}$ binding sites of the EF- hand type (i.e., helix-loop-helix arrangement) [11]. The $\mathrm{C}$-terminal EF hand contains the classical canonical $\mathrm{Ca}^{2+}$ binding motif, while the other one, located in the $\mathrm{N}$ terminal half, includes two additional amino acids and is therefore also called pseudo or S100 EF hand. As a consequence, $\mathrm{Ca}^{2+}$ binding to the individual $\mathrm{EF}$ hands occurs with different affinities, ranging from a dissociation constant $\left(K_{\mathrm{d}}\right)$ of 10 to $50 \mu \mathrm{M}$ in case of the C-terminal EF hand to a $K_{\mathrm{d}}$ between 200 and $500 \mu \mathrm{M}$ in case of the $\mathrm{N}$-terminal EF site [12]. Importantly, $\mathrm{Ca}^{2+}$ affinity is modified by posttranslational mechanisms resulting in $\mathrm{Ca}^{2+}$ binding and subsequent activation of $\mathrm{S} 100$ proteins at much lower $\mathrm{Ca}^{2+}$ concentrations [13-15].

In S100 proteins, the two EF hands are interconnected by an intermediate linker region, usually referred to as the hinge region, and the C-terminal helix-loop-helix arrangement is followed by a C-terminal extension (Fig. 1). As members of the S100 protein family differ from one another mostly in length and sequence of both the hinge region and the C-terminal extension, these sections are suggested to mediate the specific biological activity of individual proteins [11]. Interestingly, $\mathrm{Ca}^{2+}$ binding of $\mathrm{S} 100$ proteins - excepting S100A10-leads to a major conformational change characterized by the formation of a hydrophobic pocket containing residues of the hinge region and the C-terminal extension, which are thus believed to represent the major interaction sites with target proteins.

Further characteristics of proteins subsumed in the multigenic S100 family include their tendency to form noncovalent homo- or heterodimers as well as a distinctive expression pattern which follows a cell type, tissue, and development stage-specific manner and could indicate a high degree of evolutionary specification [16]. At present, S100 proteins have been identified as regulators of fundamental molecular and cellular functions such as differentiation, proliferation, hypertrophy, apoptosis, and motility in both physiological and pathophysiological

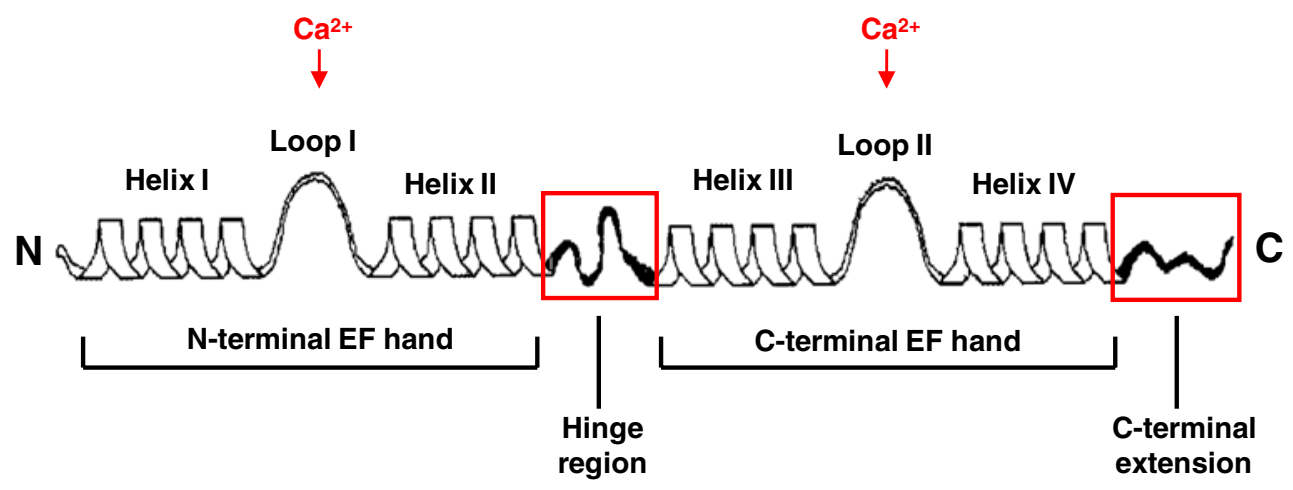

Fig. 1 Schematic depiction of the secondary structure of an S100 protein. Each $\mathrm{S} 100$ monomer consists of a repetitive $\mathrm{Ca}^{2+}$ binding EFhand motif whereas the $\mathrm{N}$-terminal (non-canonical) and $\mathrm{C}$-terminal (canonical) EF hand are connected by a linker region (hinge region).
The hinge region and C-terminal extension (boxed in red) display the greatest sequence variability among individual members of the S100 protein family. Reproduced with modifications from Donato et al. [11] 
conditions including psoriasis, rheumatoid arthritis, several neurological disorders, and a diverse list of cancers $[12,17-19]$. Based on the discovery of discriminative expression patterns in healthy and diseased hearts and vasculature, experimental in vitro and in vivo studies additionally revealed that the integrity of $\mathrm{S} 100$ proteins like S100A1, S100A4, S100A6, and S100B is a basic requirement for normal cardiovascular development and function $[8,20-37]$. Due to its ability to modulate numerous key effector proteins in cardiomyocytes and endothelial cells, S100A1 in particular has been proven to play a critical role in both cardiac performance and vascular biology.

\section{S100A1 Molecular Structure}

The S100A1 monomer with a molecular weight of $10.4 \mathrm{kDa}$ reflects the molecular architecture typical for all members of the S100 protein family [11]. In each EF hand, the $\mathrm{Ca}^{2+}$ binding loop is flanked by $\alpha$-helices, so that helices I and II enclose the N-terminal loop and helices III and IV, the C-terminal loop, respectively. Within cells, the dominating condition of S100A1 is a homodimer of two S100A1 molecules whereas the dimeric structure is favored even at picomolar monomer concentrations [12, 16, 38]. Dimerization of S100A1 occurs independently of $\mathrm{Ca}^{2+}$ binding in a symmetric and antiparallel manner and is stabilized through hydrophobic bonds between helices I and $I^{\prime}$ of each monomer (Fig. 2).

Upon $\mathrm{Ca}^{2+}$ binding to both EF hands, S100A1-alike other S100 proteins - undergoes a conformational change resulting in the exposure of a concave hydrophobic pocket [39]. Considering the fact that the pocket-forming domains, including the hinge region and the $\mathrm{C}$-terminal extension, display the greatest sequence variability among $\mathrm{S} 100$ isoforms, this pocket is considered to be involved in the $\mathrm{Ca}^{2+}$-dependent interaction with target proteins, enabling S100A1 to function as an intracellular $\mathrm{Ca}^{2+}$ sensor without apparent intrinsic catalytic activity [12]. Despite the different $\mathrm{Ca}^{2+}$ affinities of both EF hands, which is a unique feature of $\mathrm{S} 100$ proteins, $\mathrm{Ca}^{2+}$ binding of $\mathrm{S} 100 \mathrm{~A} 1$ is tightly regulated by posttranslational modifications [13-15]. NO-dependent $S$-glutathionylation of a cysteine residue located in the $\mathrm{C}$-terminal extension facilitates $\mathrm{Ca}^{2+}$ binding of both $\mathrm{EF}$ hands even at nanomolar-free $\mathrm{Ca}^{2+}$ concentrations. Additionally, recent findings suggest that S100A1 can only function as a $\mathrm{Ca}^{2+}$ sensing protein after glutathionylation of its Cys85 residue. Redox- and NO-dependent posttranslational modifications might therefore empower S100A1 to sense spatially defined short-term as well as long-term global $\mathrm{Ca}^{2+}$ oscillations in cardiac and vascular cells, whereas its $\mathrm{Ca}^{2+}$ sensing function might be regulated by the particular intracellular NO/redox equilibrium [40].

\section{S100A1 Cardiovascular Expression Pattern}

In humans, S100A1 is predominantly found in the myocardium with the highest protein levels in the left ventricle and lower concentrations in the right ventricle and the atria [41-45]. Comparative analyses revealed a similar S100A1 expression in a wide range of animals including

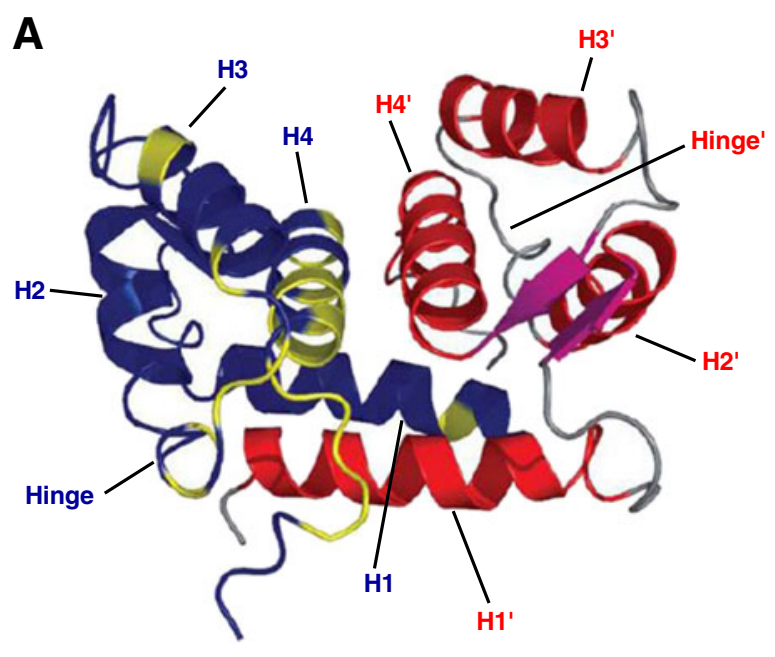

Fig. 2 The three-dimensional structure of S100A1 as determined by NMR spectroscopy. a In the apo-state, S100A1 exists as a homodimer. Dimerization occurs in an antiparallel manner and is stabilized by hydrophobic bonds between helix $\mathrm{H} 1$ and $\mathrm{H}^{\prime}$ '. b The conformational change upon $\mathrm{Ca}^{2+}$ binding to both the $\mathrm{N}$ - and $\mathrm{C}$-terminal $\mathrm{EF}$ hands

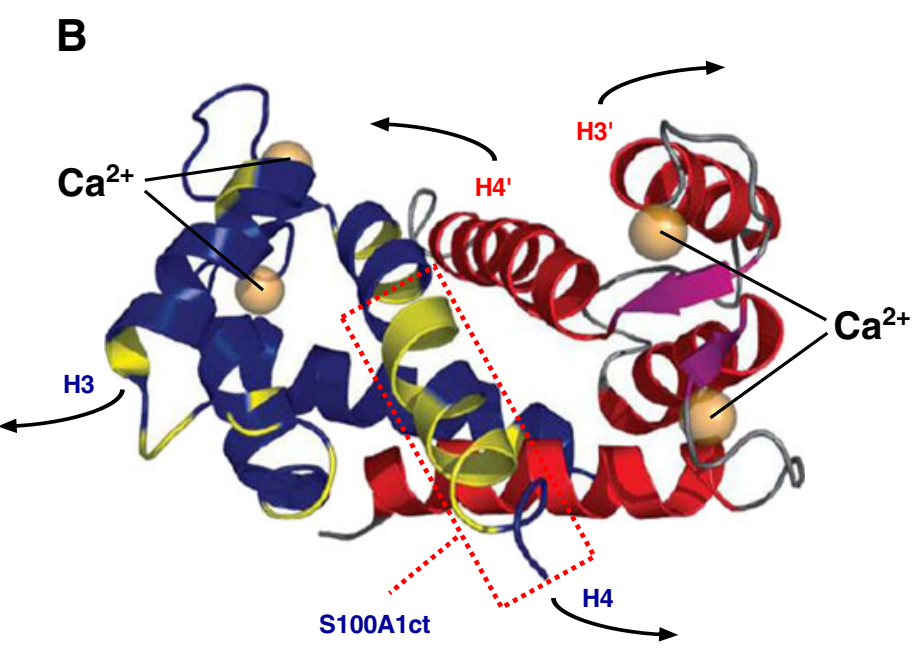

results in the exposure of hydrophobic residues that are important for the interaction with target molecules. S100A1 residues 75-94 are boxed in red (S100A1ct). Reproduced with modifications from Wright et al. [16] 
rodents and larger mammals $[42,43,46]$. In mice, cardiac S100A1 mRNA and protein levels steadily increase during heart development and reach a plateau in the postnatal state [47]. Among the cell types habitually present in the postpartum myocardium, S100A1 predominantly resides in cardiomyocytes without evidence for an expression in cardiac fibroblasts (Rohde D, unpublished results). Analysis of the S100A1 promoter in rodents identified a subset of transcription factor consensus sequences (i.e., Nkx-2.5, MEF-2, and CEF) that are well known to drive cardiacspecific expression of genes such as cardiac troponin $C$ or calsequestrin and potentially convey the cardiac-restricted expression of S100A1. Theoretical calculations derived from cell culture experiments using recombinant S100A1 for quantitative protein analysis indicated a concentration of approximately $50 \mathrm{nM}$ in the cytoplasmic compartment of adult rat and mouse ventricular cardiomyocytes (Rohde D and Voelkers M, unpublished results) [48].

Although S100A1 expression has never been detected in the vasculature of fetal mice, S100A1 mRNA and protein have recently been described in rodent and human endothelial cells from different tissues [30, 49-51]. Expression analyses in rodents revealed an approximately 50 -fold lower S100A1 protein content of adult aortic endothelial cells in comparison with cardiomyocytes (Most P, unpublished results). Against this background, both a missing expression and an S100A1 expression below the methodical detection limit could hypothetically be the case in maturing rodent vascular structures, but do not necessarily reflect expression patterns in humans.

\section{S100A1 in Cardiomyocytes}

\section{Subcellular Location and Molecular Targets}

In immunohistochemical analyses, S100A1 displayed a striated-like cytoplasmic pattern in adult cardiomyocytes, mainly co-localizing with the sarcoplasmic reticulum ATPase (SERCA2a) and the myocardial ryanodine receptor 2 (RyR2) [27, 52-55]. Further studies also yielded a partial co-localization with mitochondria in neonatal cardiomyocytes, whereas the mitochondrial $\mathrm{F}_{1}$-ATPase and the adenine nucleotide translocator (ANT) could be identified as binding targets of S100A1 [56, 57]. Moreover, the use of immuno-electron microscopy uncovered several S100A1 binding regions within the sarcomere, including the Z-line, the periphery of the M-lines, as well as the I- and A-bands, and subsequent analyses revealed the giant myofilament protein titin as a binding partner of S100A1 [58-61].

Sarcoplasmic reticulum Several studies reported a colocalization of S100A1 with the SERCA2a/phospholamban
(PLB) complex in cardiomyocytes and demonstrated a $\mathrm{Ca}^{2+}$ dependent interaction of S100A1 with both the sarcoplasmic reticulum (SR) $\mathrm{Ca}^{2+}$ ATPase and its endogenous inhibitory peptide PLB [27, 52, 53, 55]. Functional analyses in cardiomyocytes and isolated SR vesicles showed that $\mathrm{Ca}^{2+}$. dependent S100A1 binding to SERCA2a results in an increased enzymatic activity and hereby heightened SR $\mathrm{Ca}^{2+}$ uptake and enhanced SR $\mathrm{Ca}^{2+}$ load $[48,54,62]$. Similar results were obtained using a synthetic peptide composed of the S100A1 C-terminal amino acid residues 75 to 94 , indicating the crucial role of helix IV and the $\mathrm{C}$-terminal extension in the context of S100A1 $\mathrm{Ca}^{2+}$-dependent conformational change and the interaction with SERCA2a [27]. Interestingly, S100A1 seems to exert its SERCA2a regulatory activity without affecting the PLB to SERCA2a ratio or PLB phosphorylation at Ser16 or Thr17. The latter suggests that neither protein kinase A (PKA) nor $\mathrm{Ca}^{2+} /$ calmodulindependent protein kinase II (CaMKII) are involved in S100A1-SERCA2a interference.

As observed in numerous studies, S100A1 protein modulates RyR2 function under both diastolic and systolic conditions [27, 52, 63, 64]. Most et al. first demonstrated that addition of S100A1 to isolated SR vesicles resulted in diminished ${ }^{3} \mathrm{H}$-ryanodine binding to RyR2 at free $\mathrm{Ca}^{2+}$ concentrations of about $150 \mathrm{nM}$, while a significantly increased ${ }^{3} \mathrm{H}$-ryanodine-RyR2 binding occurred at $\mathrm{Ca}^{2+}$ concentrations greater than $300 \mathrm{nM}$ [27]. The relevance of this $\mathrm{Ca}^{2+}$-dependent biphasic influence of S100A1 on RyR2 could be approved by multiple subsequent analyses [48, 62-64]. Hypothesizing a reduced RyR2 open probability at diastolic cytoplasmic $\mathrm{Ca}^{2+}$ levels, Voelkers et al. showed that S100A1 in fact decreases the frequency and amplitude of elementary SR $\mathrm{Ca}^{2+}$ events $\left(\mathrm{Ca}^{2+}\right.$ sparks $)$ and thereby reduces the $\mathrm{SR} \mathrm{Ca}^{2+}$ leak in quiescent cardiomyocytes [63]. Moreover, a recent study confirmed that S100A1 increases fractional SR $\mathrm{Ca}^{2+}$ release in voltageclamped rabbit cardiomyocytes, suggesting that S100A1 enhances the excitation-contraction coupling gain under systolic conditions [52].

The differential effects of S100A1 on RyR2 activity could be interpreted as the result of several binding sites. Indeed, mapping analyses revealed at least three distinct S100A1 binding domains within the cytoplasmic portion of the skeletal muscle ryanodine receptor isoform (RyR1), including the calmodulin binding domain of RyR1 [65]. Additionally, current investigations indicate that the S100A1-RyR2 binding reaction is $\mathrm{Ca}^{2+}$ dependent and includes - analogous to SERCA2a - the S100A1 C-terminal amino acid residues 75 to 94 [64]. However, S100A1's interaction sites with RyR2 remain to be characterized in detail.

Taken together, several studies investigated the impact of S100A1 on SR $\mathrm{Ca}^{2+}$ homeostasis and consistently found 
that S100A1 augments both the systolic $\mathrm{Ca}^{2+}$ induced $\mathrm{Ca}^{2+}$ release and the diastolic SR $\mathrm{Ca}^{2+}$ uptake by modulating SERCA2a and RyR2 activity. In doing so, S100A1together with other regulative proteins - might essentially contribute to an intact SR function and a physiological $\mathrm{Ca}^{2+}$ cycling in cardiomyocytes.

Mitochondria The combined use of confocal and immunoelectron microscopy leads to the detection of S100A1 located in the mitochondria of cardiomyocytes [56, 57]. Boerries et al. first described a $\mathrm{Ca}^{2+}$-dependent interaction of S100A1 with the mitochondrial $\mathrm{F}_{1}$-ATPase, which is of crucial importance for ATP synthesis and energy supply by oxidative phosphorylation in animal and human cells [56, 66]. The same study provided further evidence for a direct stimulation of ATP synthase activity by S100A1, suggesting a fundamental role of S100A1 in the regulation of cardiac energy homeostasis. Accordingly, a significant lower mitochondrial ATPase activity and decreased ATP levels were found in S100A1 deficient cardiomyocytes, whereas a normalization of S100A1 abundance in failing cardiomyocytes restored ATP levels to normal values [27]. In addition to the $\mathrm{F}_{1}$-ATPase, the ANT could be identified as another binding target of S100A1 in mitochondria, indicating a possible involvement of S100A1 in the ADP/ATP exchange between the mitochondrial matrix and the cytoplasm [56]. As recent analyses brought to light that S100A1 apparently increases mitochondrial $\mathrm{Ca}^{2+}$ uptake (Boerries M, unpublished results), it is tempting to speculate that S100A1 interaction with mitochondrial targets is not only an integral part of cardiac muscle energy metabolism but also a regulator of cardiomyocyte apoptosis [67]. However, additional studies are needed to elucidate the impact of S100A1 on mitochondrial metabolism and the interplay between the SR and mitochondria in cardiomyocytes.

Sarcomere Besides SR and mitochondria, S100A1 has also been detected at several sites within the sarcomere [42, 43, $60,61]$. Yamasaki et al. first provided comprehensive evidence for S100A1 as a binding partner of the giant myofilament protein titin and identified three distinctive S100A1 interaction sites within its force-generating region [61]. In this context, $\mathrm{Ca}^{2+}$-dependent S100A1 interaction with the PEVK subdomain of titin was reported to result in improved sarcomeric compliance by reducing the force that arises as F-actin slides relative to the PEVK domain. Based on their results, Granzier and colleagues hypothesized that S100A1-titin interaction represents a regulatory mechanism which leads to diminished pre-contractile passive tension in cardiomyocytes [61, 68]. Interestingly, it has previously been shown that $\mathrm{S} 100 \mathrm{~A} 1$ reduces myofilament $\mathrm{Ca}^{2+}$ sensitivity and cooperativity without affecting troponin I phosphorylation or maximal force development [54]. Taken together, $\mathrm{Ca}^{2+}$-dependent S100A1 interplay with components of the sarcomere might facilitate diastolic cardiomyocyte function by SR-independent mechanisms, whereby the precise molecular and functional relationships remain to be characterized.

Impact on $\mathrm{Ca}^{2+}$ Handling and Contractile Performance

Numerous studies have shown that overexpression of S100A1 results in increased $\mathrm{Ca}^{2+}$ transients and enhanced contractile performance of field-stimulated mice, rat, and rabbit ventricular cardiomyocytes [27, 54, 62, 69]. Enhancing intracellular S100A1 levels by application of recombinant S100A1 protein via patch pipette similarly augmented systolic SR $\mathrm{Ca}^{2+}$ release and amplified the excitation-contraction coupling gain in voltage-clamped rabbit cardiomyocytes [52]. These observations most likely reflect the regulatory effects of S100A1 interaction with target proteins directly involved in cardiomyocyte $\mathrm{Ca}^{2+}$ handling and contractile properties. Strikingly, increased S100A1 protein levels have a combined impact on both diastolic and systolic cardiomyocyte performance (Fig. 3).

In diastole, S100A1-mediated enhancement of SERCA2a activity and a diminished frequency and amplitude of SR $\mathrm{Ca}^{2+}$ sparks account for a heightened SR $\mathrm{Ca}^{2+}$ load and an accelerated cardiomyocyte relaxation $[52,53,55$, 63]. Additionally, eased myofilament $\mathrm{Ca}^{2+}$ dissociation and a reduced titin-based pre-contractile passive tension further contribute to an improved cardiomyocyte diastolic function $[61,68]$. In systole, on the other hand, an S100A1-mediated increase in $\mathrm{SR} \mathrm{Ca}^{2+}$ load in concert with augmented RyR2 opening and fractional SR $\mathrm{Ca}^{2+}$ release represents a powerful mechanism to boost $\mathrm{Ca}^{2+}$ transients and support systolic mechanical force development [48, 52, 54, 62]. However, the underlying molecular mechanism by which S100A1 might alter systolic SR $\mathrm{Ca}^{2+}$ release, i.e., by altered RyR2 gating remains to be determined. Finally, enhanced ATP availability caused by an S100A1-mediated increase in mitochondrial ATPase activity might enable cardiomyocytes to better match $\mathrm{Ca}^{2+}$-dependent energetic demands $[56,57]$.

Expression analyses yielded that S100A1-mediated effects on $\mathrm{Ca}^{2+}$ cycling and contractile performance do not result from an altered abundance of SR proteins [54]. Furthermore, it has been shown that S100A1 influences neither the L-type $\mathrm{Ca}^{2+}$ current nor the $\mathrm{Na}^{+}-\mathrm{Ca}^{2+}$ exchanger (NCX) in adult cardiomyocytes [52, 63]. Moreover, S100A1-overexpressing cardiomyocytes displayed their hypercontractile phenotype on top of and independent from $\beta$-adrenergic receptor $(\beta A R)$ stimulation with unchanged $\beta$ AR downstream signaling and related protein expression 
Fig. 3 Proposed model for S100A1 molecular interactions in cardiomyocytes. a During excitation-contraction coupling, voltage-dependent opening of the L-type $\mathrm{Ca}^{2+}$ channel (LTCC) leads to a transsarcolemmal $\mathrm{Ca}^{2+}$ entry that triggers sarcoplasmic reticulum $(S R)$ $\mathrm{Ca}^{2+}$ release via ryanodine receptor $2(R y R 2)$. Increased sarcoplasmic $\mathrm{Ca}^{2+}$ levels eventually result in myofilament cross-bridge cycling and mechanical force development. During diastole, resequestration of sarcoplasmic $\mathrm{Ca}^{2+}$ into the $\mathrm{SR}$ is operated by the $\mathrm{SR} \mathrm{Ca}^{2+}$ ATPase (SERCA). To keep steady-state conditions, the NCX excerts transsarcolemmal $\mathrm{Ca}^{2+}$ extrusion in balance with LTCC-conducted $\mathrm{Ca}^{2+}$ entry. b S100A1 interacts with both RyR2 and SERCA and is present at mitochondria and myofilaments. Increased S100A1 protein levels enhance systolic SR $\mathrm{Ca}^{2+}$ release via RyR2 without influencing LTCC-conveyed $\mathrm{Ca}^{2+}$ entry. Augmented systolic SR $\mathrm{Ca}^{2+}$ release is compensated by elevated SERCA activity and diminished SR $\mathrm{Ca}^{2+}$ leakage during diastole, altogether improving SR $\mathrm{Ca}^{2+}$ cycling and mechanical force generation. Additionally, S100A1 interaction with mitochondrial $\mathrm{F}_{1}$-ATPase leads to increased ATP synthesis and enhanced energy supply. Finally, myofilament pre-contractile passive tension is reduced by S100A1 interference with titin-actin interplay in the sarcomere
A
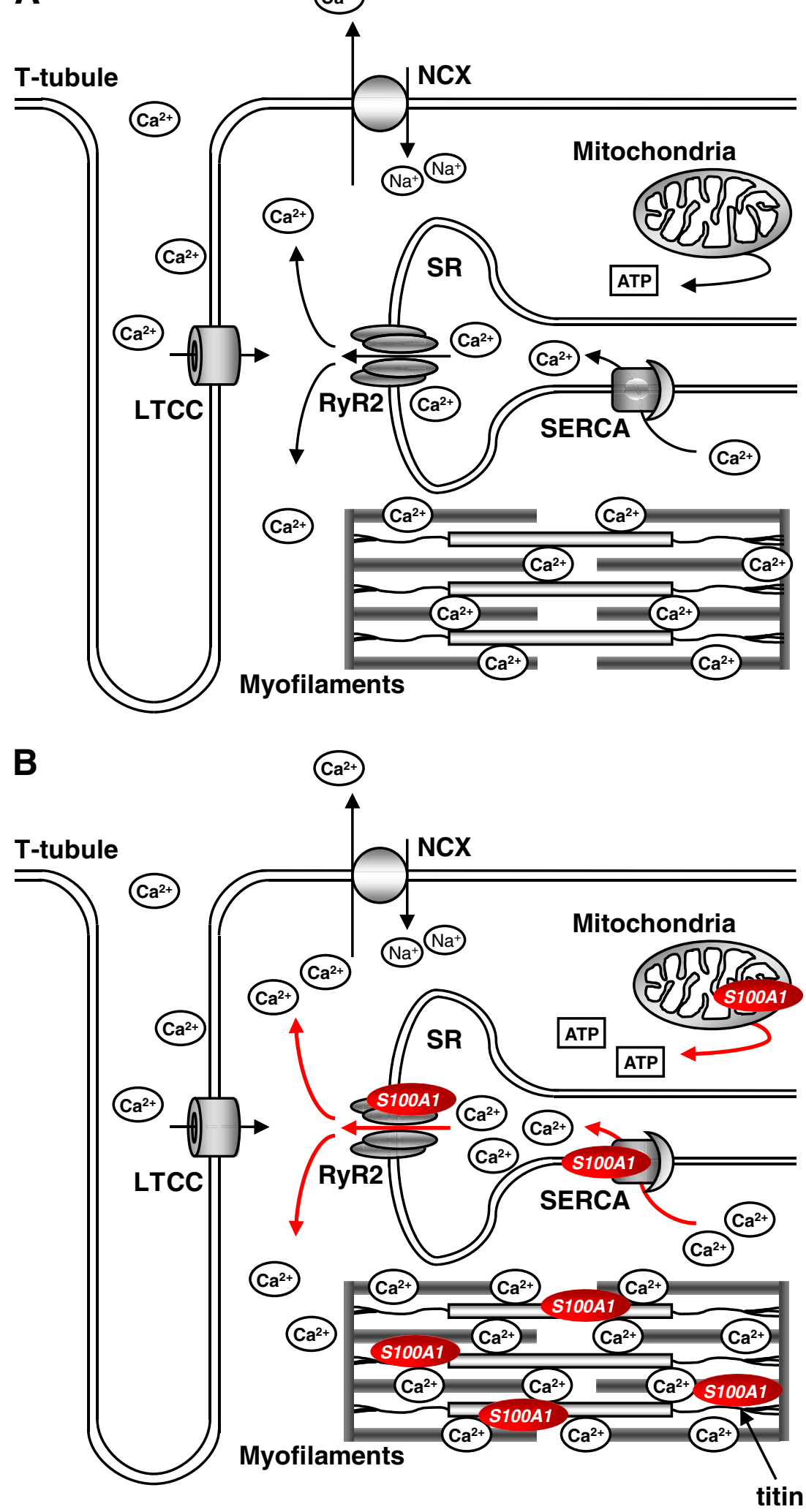
$[27,54,69-71]$. This is of particular interest for cardiovascular physiology as chronic stimulation of $\beta$ AR signaling and its downstream effectors such as PKA eventually leads to cardiac hypertrophy and heart failure [72, 73].

In summary, S100A1 specifically targets cardiomyocyte $\mathrm{Ca}^{2+}$ handling by improving SR $\mathrm{Ca}^{2+}$ uptake as well as SR $\mathrm{Ca}^{2+}$ leakage during diastole and $\mathrm{Ca}^{2+}$ release during systole. In concert with reduced myofilament $\mathrm{Ca}^{2+}$ cooperativity and pre-contractile passive tension, these molecular mechanisms synergistically facilitate an optimized diastolic and systolic contractile performance of cardiomyocytes. Additionally, S100A1 mitochondrial actions might reflect a mechanism to attune $\mathrm{Ca}^{2+}$-dependent energetic demands to oxidative energy production. Although our understanding of how S100A1 modulates cardiomyocyte metabolism is still incomplete and warrants further investigation, S100A1 could represent a promising therapeutic target in acute and chronic heart failure.

\section{S100A1 in Endothelial Cells: Molecular Targets and Regulatory Function}

Lefranc et al. first described S100A1 expression in endothelial cells (ECs) [51]. The following analyses revealed S100A1 expression in rodent and human capillary, aortic, femoral, pulmonary, and coronary artery endothelial cells [30, 49-51]. In cultured aortic endothelial cells, S100A1 is mainly dispersed in a granular pattern throughout the cytoplasm with perinuclear enrichment [30]. The use of immunohistochemistry and confocal microscopy disclosed the presence of S100A1 at the endoplasmic reticulum (ER) co-localizing with both endothelial SERCA2 and the inositol-1,4,5-triphosphate receptor $\left(\mathrm{IP}_{3} \mathrm{R}\right)$. Most recently, co-immunoprecipitation of endothelial nitric oxide synthase (eNOS) and S100A1 from EC lysates indicated a direct $\mathrm{Ca}^{2+}$-dependent binding of S100A1 to eNOS (Most P, unpublished results).

Just like its interaction with SERCA2a and RyR2 in cardiomyocytes, S100A1 seems to target SERCA2 and $\mathrm{IP}_{3} \mathrm{R}$ as key effectors of ER $\mathrm{Ca}^{2+}$ uptake and release in endothelial cells [74]. Indeed, Pleger et al. demonstrated an abnormal agonist-induced intracellular $\mathrm{Ca}^{2+}$ rise in ECs with no or depleted levels of S100A1 [30]. Thus, ECs isolated from $\mathrm{S} 100 \mathrm{~A} 1$ knockout mice $\left(\mathrm{SKO}^{--}\right)$exhibited reduced $\mathrm{Ca}^{2+}$ transients in response to acetylcholine stimulation. Consistently, siRNA-mediated S100A1 downregulation resulted in a significantly decreased bradykinininduced cytoplasmic $\mathrm{Ca}^{2+}$ peak formation. Supporting a critical role for $\mathrm{S} 100 \mathrm{~A} 1$ in $\mathrm{IP}_{3}$-mediated $\mathrm{Ca}^{2+}$ signaling in $\mathrm{ECs}$, adenoviral $\mathrm{S} 100 \mathrm{~A} 1$ expression in $\mathrm{SKO}^{-/-}$cells led to a normalization of $\mathrm{Ca}^{2+}$ transients in response to acetylcholine and bradykinin.
Against this backdrop, it was tempting to hypothesize an effect of S100A1 on NO homeostasis in ECs as NO production is regulated by the $\mathrm{Ca}^{2+}$-dependent binding of calmodulin to eNOS. Pleger et al. as well as Desjardins et al. showed that ECs isolated from $\mathrm{SKO}^{-/-}$mice exhibit an impaired baseline NO production with an attenuated responsiveness to acetylcholine, bradykinin, and thrombin in comparison with wild-type (WT) controls [29, 49]. Similar results were obtained from S100A1 siRNA-treated ECs with significantly decreased S100A1 protein levels. Interestingly, differences in NO generation occurred at comparable expression levels of eNOS in ECs from $\mathrm{SKO}^{-/-}$ and WT animals. In line with these observations, S100A1 overexpression in human coronary artery endothelial cells resulted in an increased NO release upon stimulation with acetylcholine, whereas this effect was abrogated in the presence of 2-amino-4-phosphonobutyrate, an inhibitor of the $\mathrm{IP}_{3} \mathrm{R}$-mediated ER $\mathrm{Ca}^{2+}$ release [29].

Summing up, these results provide evidence for a regulatory role of S100A1 in EC NO homeostasis (Fig. 4). S100A1 deficiency presumably interrupts normal muscarinergic and kininergic receptor-mediated eNOS activation at the level of $\mathrm{IP}_{3}$-triggered $\mathrm{ER} \mathrm{Ca}^{2+}$ release, which potentially leads to diminished $\mathrm{Ca}^{2+}$-dependent binding of calmodulin to eNOS. Recent observations from Most et al. additionally indicate a direct molecular interaction of S100A1 with eNOS, which could contribute to the modulatory effect of S100A1 on NO generation [50] (Most P, unpublished results). However, it remains to be established whether S100A1 exclusively targets ER $\mathrm{Ca}^{2+}$ handling and eNOS regulation or affects alternative regulatory pathways in ECs.

\section{S100A1 in Cardiac Disease}

\section{Pathophysiological Implications}

The first clinical interest in S100A1 has been sparked due to its altered expression in diseased myocardium. Remppis et al. first demonstrated that chronically dysfunctional human myocardium is characterized by progressively diminished S100A1 protein levels that correlate inversely with the clinical severity of congestive heart failure [44]. Subsequent analyses in a variety of animal heart failure models, including rodents, rabbit, and pig, provided further evidence for decreased cardiac S100A1 protein levels as a molecular signature of failing myocardium in vivo [27, 48, $70,71,75,76]$. Most et al. were able to recapitulate this notion in vitro by incubating cardiomyocytes with various hypertrophic stimuli [48]. Indeed, chronic stimulation of neonatal rat cardiomyocytes with endothelin 1 , phenylephrine , and transforming growth factor $\beta$ resulted in a dose- 
Fig. 4 Proposed model for S100A1 molecular interactions in endothelial cells. a Muscarinergic $\mathrm{G}_{\mathrm{q}}$-protein coupled receptor ( $m A$ chR) stimulation results in an inositol-1,4,5-triphosphate $\left(\mathrm{IP}_{3}\right)$-mediated ER $\mathrm{Ca}^{2+}$ release. Increased cytoplasmic $\mathrm{Ca}^{2+}$ levels induce enhanced $\mathrm{Ca}^{2+}$-dependent calmodulin $(\mathrm{CaM})$ binding to endothelial nitric oxide synthase (eNOS), eventually leading to an augmented NO production. b S100A1 interacts with both $\mathrm{IP}_{3}$-receptor $\left(I P_{3} R\right)$ and SERCA. Increased $\mathrm{S} 100 \mathrm{~A} 1$ protein levels enhance $\mathrm{IP}_{3}$-mediated ER Ca ${ }^{2+}$ release, inducing a heightened $\mathrm{Ca}^{2+}$-dependent CaM-mediated stimulation of eNOS activity. Additionally, direct S100A1 binding to eNOS potentially further increases $\mathrm{NO}$ generation in endothelial cells
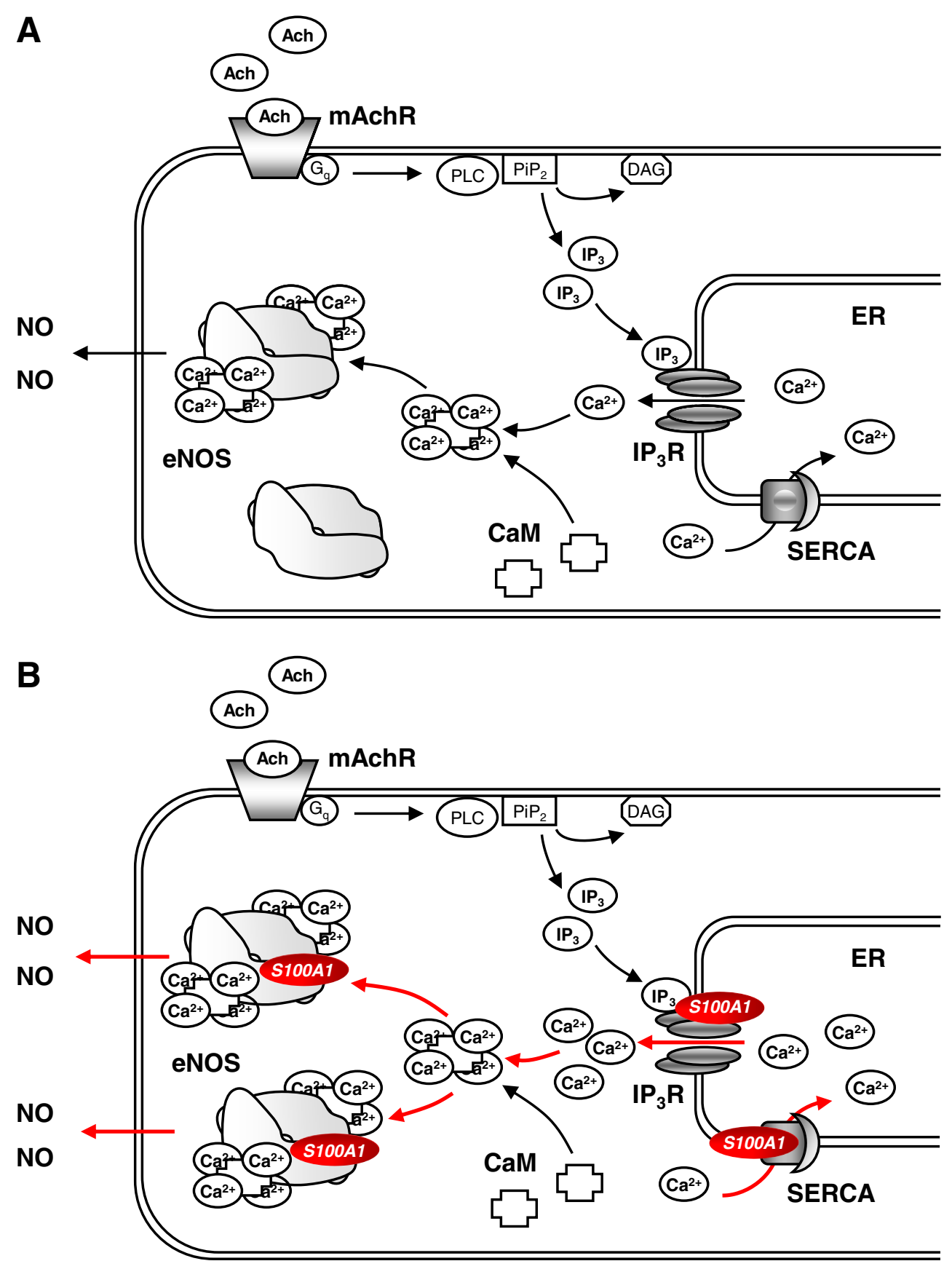

and time-dependent progressive decrease in S100A1 mRNA and protein. As Kiewitz et al. identified an activator protein 1 (AP-1) binding site within the murine S100A1 promoter sequence, $\mathrm{G}_{\mathrm{q}}$-protein coupled receptor downstream signaling, which is known to include AP-1, might directly operate the transcriptional inhibition of S100A1 [47]. In light of its low abundance in developing fetal hearts, S100A1 downregulation could furthermore be considered as a part of fetal gene reprogramming in maladaptive cardiomyocyte hypertrophy.

The discovery of reduced S100A1 protein levels in failing myocardium raised the question whether S100A1 is directly involved in the pathophysiological molecular mechanisms underlying the clinical development of heart failure. Interestingly, homozygous S100A1 knockout mice $\left(\mathrm{SKO}^{-/-}\right)$showed unaltered heart rate and cardiac function under baseline conditions, but exhibited enhanced susceptibility and accelerated functional deterioration in response to $\beta$ AR stimulation, pressure overload, and ischemic injury $[48,49,77]$. $\mathrm{SKO}^{-1-}$ mice subjected to transaortic constriction (TAC) for instance displayed significant contractile impairment and transition towards heart failure 3 weeks after surgery, while WT animals developed a functionally compensated state [77]. Surprisingly, heterozygous S100A1 
knockout mice $\left(\mathrm{SKO}^{-/+}\right)$, with approximately half the cardiac S100A1 protein abundance of WT animals, initially also presented an impaired response to $\beta A R$ stimulation but maintained normal contractility in response to chronic pressure overload. Expression analyses revealed that $\mathrm{SKO}^{-/+}$mice subjected to TAC show an adaptive left ventricular upregulation of S100A1 to protein levels found in WT animals, potentially enabling them to establish a compensated state in the long term [77]. In line with these results, myocardial infarction (MI) in $\mathrm{SKO}^{-/-}$mice resulted in accelerated transition to heart failure, enhanced cardiac remodeling, increased cardiomyocyte apoptosis, and excessive mortality in comparison with WT controls [48, 49]. Additionally, hearts from infarcted $\mathrm{SKO}^{-/}$animals displayed $\beta A R$ signaling defects and impaired SR $\mathrm{Ca}^{2+}$ handling significantly earlier than organs from WT mice [48].

Taken together, these data provide evidence that S100A1 represents an essential prerequisite for cardiac adaption to chronic hemodynamic stress and ischemic damage. Considering the hypercontractile phenotype of S100A1overexpressing cardiomyocytes, different in vivo strategies, including transgenic mice and S100A1 gene delivery, have been used to support this concept [27, 48, 62, 71]. Compared with WT controls, transgenic mice with a cardiac-restricted overexpression of S100A1 (STG) presented a markedly augmented cardiac function that remained elevated in response to $\beta$ AR stimulation [48, 62]. Concomitantly, both SR $\mathrm{Ca}^{2+}$ content and $\mathrm{Ca}^{2+}$ induced $\mathrm{SR} \mathrm{Ca}^{2+}$ release were significantly increased in ventricular cardiomyocytes isolated from STG animals. Remarkably, STG mice, compared with $\mathrm{SKO}^{--}$and WT animals, maintained almost normal left ventricular function and exhibited clearly reduced cardiac remodeling and cardiomyocyte apoptosis as well as an improved survival in response to myocardial infarction [48]. Similar results were obtained from rats with an adenoviral transgenemediated cardiac overexpression of S100A1, indicating the feasibility of a cardiac S100A1 gene transfer in vivo [71].

All in all, the acute and chronic heart failure animal models investigated clearly mirror the impact of S100A1 on cardiomyocyte $\mathrm{Ca}^{2+}$ handling and contractile performance studied in vitro. Whether the improved functional outcome and superior survival of S100A1-overexpressing animals in response to pressure overload and myocardial infarction is solely caused by the known molecular interactions of S100A1 in cardiomyocytes, however, seems to be questionable. Given that S100A1, like other S100 proteins, also exhibits extracellular functions [78-82], the formerly reported myocardial damage-associated release of S100A1 could be of special importance in this context [36, 83]. Besides its anti-apoptotic effect on neonatal cardiomyocytes [82], Rohde et al. most recently demonstrated a paracrine influence of extracellular S100A1 on adult rat cardiac fibroblasts, potentially providing an additional molecular mechanism underlying the improved post-MI prognosis of S100A1-overexpressing mice [84]. Nevertheless, the pathophysiological significance of S100A1 released from injured cardiomyocytes still needs to be investigated.

\section{S100A1 Therapy of Diseased Myocardium}

The results obtained from genetically altered mice comprehensively point out the critical role of S100A1 in the field of cardiac functional performance and the development of heart failure in response to pressure overload and ischemic injury. Together with an altered expression in human failing myocardium and the identified molecular interactions in cardiomyocytes, these data provide a strong rationale to propose S100A1 as a novel therapeutic target for acute and chronic cardiac dysfunction.

Adenoviral-based S100A1 gene delivery in failing rat ventricular cardiomyocytes normalized S100A1 protein abundance and restored physiological contractile performance yielding first proof-of-concept for the therapeutic potential of S100A1 gene therapy [27, 70, 71]. Detailed analyses of SR $\mathrm{Ca}^{2+}$ handling in failing cardiomyocytes displayed re-established $\mathrm{Ca}^{2+}$ transients, normalized SR $\mathrm{Ca}^{2+}$ load, and decreased diastolic SR $\mathrm{Ca}^{2+}$ leakage in response to S100A1 treatment [27]. Additionally, Voelkers et al. most recently demonstrated that S100A1 gene delivery can actually prevent $\beta A R$ stimulation-triggered diastolic SR $\mathrm{Ca}^{2+}$ leakage in adult cardiomyocytes [85]. This finding is of particular clinical interest as a reduction of spontaneous $\mathrm{SR} \mathrm{Ca}^{2+}$ release during diastole might reduce both incidence and severity of cardiomyocyte after depolarization-induced cardiac arrhythmias. In this way, S100A1 overexpression could contribute to the previously reported improved post-MI survival of STG mice.

Building on these experiments, in vivo approaches of S100A1 gene therapy were pursued in rodents and pigs $[27$, 70, 71, 76]. Both adenoviral- and adeno-associated virus (AAV)-based intracoronary S100A1 gene delivery to failing rat hearts resulted in reversed maladaptive myocardial remodeling and a rescue of contractile performance [27, 71]. Moreover, another study most recently provided proof of therapeutic effectiveness for S100A1 gene therapy in an experimental pig heart failure model [76]. Here, domestic pigs were subjected to myocardial infarction and were undergone a retroinfusion-facilitated delivery of AAV9S100A1 to the anterior coronary vein 2 weeks later. In postMI pigs, AAV9-S100A1 application led to protection from progressive hemodynamic deterioration and significant improvement of cardiac function, indicating a long-term rescue of heart failure. Interestingly, S100A1-treated animals, in contrast to untreated controls, developed heart rate 
normalization in response to therapeutic intervention, reflecting an abrogation of the sympathetic overdrive postMI [76].

However, S100A1-based therapeutic interventions in cardiac disease are not limited to myocardial gene delivery and could potentially also be employed in the emerging fields of tissue engineering and cell therapy. Indeed, engineered heart tissue (EHT) constructed from neonatal rat cardiomyocytes displayed enhanced contractile performance under baseline conditions and in response to $\beta \mathrm{AR}$ stimulation after adenoviral overexpression of S100A1 [86]. S100A1 gene delivery might therefore represent a promising strategy to strengthen EHT and to heighten its therapeutic effectiveness, which could open new ways of engineered tissue-based treatment options. Moreover, it is tempting to speculate that progenitor cells with the ability to regenerate cardiomyocytes could benefit from increased S100A1 protein levels in the sense of superior contractile properties and enhanced resistance against pathological myocardial remodeling.

\section{S100A1 in Vascular Disease: Impact on Vascular Tone and Therapeutic Potential}

Given the modulatory function of S100A1 in EC NO homeostasis, the first evidence for the crucial pathophysiological significance of this finding emerged from experiments on arterial vessels isolated from $\mathrm{SKO}^{-/-}$ mice. Pleger et al. and Desjardins et al. comprehensively demonstrated that S100A1 deficiency is associated with a significantly diminished relaxation in response to acetylcholine in thoracic aorta and mesenteric artery segments $[30,49,50]$. Interestingly, no differences in vasodilatation were observed between vessels from $\mathrm{SKO}^{-1-}$ and WT animals upon incubation with the NO donor sodium nitroprusside and the $\beta \mathrm{AR}$ agonist isoproterenol [30]. Although a previous study described an interaction of S100 proteins with the calmodulin binding protein caldesmon in smooth muscle cells [87], these data clearly point out that a decreased muscarinergic receptorstimulated relaxation of vessels lacking S100A1 can be attributed to a diminished EC NO production. Further supporting this notion, distinctions measured in response to acetylcholine between mesenteric artery segments from $\mathrm{SKO}^{-/}$and WT mice were abolished in the presence of the eNOS inhibitor L- $N^{\mathrm{G}}$-nitroarginine methyl ester [49].

Considering these pathophysiological relationships, the hemodynamic characterization of S100A1-deficient mice appeared to be of particular interest. Indeed, Pleger et al. described significantly elevated systolic and diastolic blood pressure values in conscious $\mathrm{SKO}^{-/-}$animals in comparison with WT littermates, whereas heart rate levels were similar in both groups [30]. In line with the in vitro results, $\mathrm{SKO}^{-/-}$ mice in contrast to WT animals showed no hypotensive response to systemic bradykinin application [30]. Moreover, Desjardins et al. most recently reported that lack of S100A1 in mice confers a gender-dependent hypertensive phenotype [49]. Surprisingly, analyses revealed significantly higher mean arterial blood pressure values in male than in female $\mathrm{SKO}^{-/-}$mice, potentially indicating hormonal differences as a confounding factor. Following myocardial infarction, male $\mathrm{SKO}^{--}$animals also displayed a reduced survival in comparison with female S100A1-deficient mice, suggesting prognostic relevance for the gender-specific extent of arterial hypertension in $\mathrm{SKO}^{-/-}$mice [49]. Thus, chronically increased cardiac afterload could contribute to the excessive post-MI mortality of $\mathrm{SKO}^{--}$mice.

Taken together, these data apparently indicate that S100A1 represents a basic prerequisite for EC NO generation and intact vascular biology. However, further studies are necessary to allow a sound understanding of S100A1's interactions in the vasculature. Thus, the relevance of S100A1 expressed in smooth muscle cells remains to be elucidated [51, 88, 89]. Nevertheless, the detection of diminished S100A1 protein levels in ECs isolated from failing rat hearts points out the close relationship and common endpoints of cardiac and vascular disease [30]. Considering the growing evidence that a dysbalanced $\mathrm{NO} /$ redox equilibrium substantially accounts for the abnormal cardiac and vascular phenotypes found in the failing cardiovascular system [40], targeting endothelial S100A1 could provide a novel therapeutic strategy for vascular disorders.

\section{Conclusions and Clinical Perspective}

To improve human health, the scientific unmasking of novel molecular mechanisms has to be translated into clinical practice. In the case of S100A1, the clinical discovery of diminished protein abundance in failing human myocardium initiated a challenging investigation of its molecular interactions and pathophysiological relevance (previously reviewed in $[26,28,50,64,74,90]$ ). At present, more than a decade later, multiple molecular interactions, regulatory functions, and pathophysiological implications of S100A1 have been identified in cardiomyocytes and ECs, making S100A1 a promising multifaceted therapeutic target in cardiovascular disease.

In the field of heart failure, several translational studies already provide evidence for the beneficial long-term effects of S100A1 gene therapy in small and large animal models $[27,48,62,70,71,76]$. Due to their striking anatomic and physiological comparability with humans, a recent study in post-MI pigs, demonstrating reversed 
myocardial remodeling and functional rescue of cardiac performance by means of S100Al gene delivery to cardiomyocytes, might help to further progress towards first clinical safety trials in humans [76]. Additionally, manipulating S100A1 expression in ECs could potentially extend the spectrum of clinical indications for S100A1based therapeutical interventions to vascular disorders like hypertension and peripheral and coronary artery disease.

However, before a human application of S100A1 gene therapy appears to be reasonable, a careful evaluation of its safety profile, including its effects on cardiac energetics and arrhythmias as well as its compatibility with established pharmacological and device-based therapies, needs to be investigated in large-animal models. Thus, although there is still a long way to go, S100A1-targeted therapies might give hope to chronically ill patients and help to fight the growing burden of cardiovascular disease in the future.

Acknowledgements This study was supported by grants from the National Institutes of Health (R01 HL092130-01 and HL092130-02S1 to Most P), Deutsche Forschungsgemeinschaft (562/1-1 to Most P and VO1659/1-1 to Voelkers M), and Bundesministerium für Bildung und Forschung (01GUO572 to Most P). T. G. Parker received funding from the Canadian Institutes of Health Research and the Heart and Stroke Foundation of Ontario.

Open Access This article is distributed under the terms of the Creative Commons Attribution Noncommercial License which permits any noncommercial use, distribution, and reproduction in any medium, provided the original author(s) and source are credited.

\section{References}

1. World Health Organization (2009). Fact Sheet No 317. Geneva: WHO.

2. Lloyd-Jones, D., et al. (2010). Heart disease and stroke statistics2010 update: A report from the American Heart Association. Circulation, 121(7), e46-e215.

3. American Heart Association (2010). Cardiovascular disease statistics. Dallas: AHA.

4. Dickstein, K., et al. (2008). ESC Guidelines for the diagnosis and treatment of acute and chronic heart failure 2008: the Task Force for the Diagnosis and Treatment of Acute and Chronic Heart Failure 2008 of the European Society of Cardiology. Developed in collaboration with the Heart Failure Association of the ESC (HFA) and endorsed by the European Society of Intensive Care Medicine (ESICM). European Heart Journal, 29 (19), 2388-2442.

5. McCann, A. B., \& Jaff, M. R. (2009). Treatment strategies for peripheral artery disease. Expert Opinion on Pharmacotherapy, 10 (10), 1571-1586.

6. Stewart, S., et al. (2001). More 'malignant' than cancer? Five-year survival following a first admission for heart failure. European Journal of Heart Failure, 3(3), 315-322.

7. Donato, R. (1999). Functional roles of S100 proteins, calciumbinding proteins of the EF-hand type. Biochimica et Biophysica Acta, 1450(3), 191-231.

8. Marenholz, I., Heizmann, C. W., \& Fritz, G. (2004). S100 proteins in mouse and man: From evolution to function and pathology (including an update of the nomenclature). Biochemical and Biophysical Research Communications, 322(4), 1111-1122.

9. Moore, B. W. (1965). A soluble protein characteristic of the nervous system. Biochemical and Biophysical Research Communications, 19(6), 739-744.

10. Schafer, B. W., et al. (1995). Isolation of a YAC clone covering a cluster of nine S100 genes on human chromosome 1q21: Rationale for a new nomenclature of the S100 calcium-binding protein family. Genomics, 25(3), 638-643.

11. Donato, R. (2003). Intracellular and extracellular roles of S100 proteins. Microscopy Research and Technique, 60(6), 540-551.

12. Zimmer, D. B., Wright Sadosky, P., \& Weber, D. J. (2003). Molecular mechanisms of S100-target protein interactions. Microscopy Research and Technique, 60(6), 552-559.

13. Goch, G., et al. (2005). Affinity of S100A1 protein for calcium increases dramatically upon glutathionylation. The FEBS Journal, 272(10), 2557-2565.

14. Zhukov, I., Ejchart, A., \& Bierzynski, A. (2008). Structural and motional changes induced in apo-S100A1 protein by the disulfide formation between its Cys 85 residue and beta-mercaptoethanol. Biochemistry, 47(2), 640-650.

15. Zhukova, L., et al. (2004). Redox modifications of the Cterminal cysteine residue cause structural changes in S100A1 and S100B proteins. Biochimica et Biophysica Acta, 1742(1-3), 191-201.

16. Wright, N. T., et al. (2005). The three-dimensional solution structure of $\mathrm{Ca}(2+)$-bound S100A1 as determined by NMR spectroscopy. Journal of Molecular Biology, 353(2), 410-426.

17. Heizmann, C. W., Fritz, G., \& Schafer, B. W. (2002). S100 proteins: structure, functions and pathology. Frontiers in Bioscience, 7, d1356-d1368.

18. Salama, I., et al. (2008). A review of the S100 proteins in cancer. European Journal of Surgical Oncology, 34(4), 357-364.

19. Zimmer, D. B., et al. (2005). S100-mediated signal transduction in the nervous system and neurological diseases. Cellular and Molecular Biology (Noisy-le-grand), 51(2), 201-214.

20. Boyd, J. H., et al. (2008). S100A8 and S100A9 mediate endotoxin-induced cardiomyocyte dysfunction via the receptor for advanced glycation end products. Circulation Research, 102 (10), 1239-1246.

21. Ehlermann, P., et al. (2006). Increased proinflammatory endothelial response to S100A8/A9 after preactivation through advanced glycation end products. Cardiovascular Diabetology, 5, 6.

22. Foell, D., et al. (2003). S100A12 (EN-RAGE) in monitoring Kawasaki disease. Lancet, 361(9365), 1270-1272.

23. Inamoto, S., et al. (2000). Isoproterenol-induced myocardial injury resulting in altered $\mathrm{S} 100 \mathrm{~A} 4$ and $\mathrm{S} 100 \mathrm{~A} 11$ protein expression in the rat. Pathology International, 50(6), 480-485.

24. Mazzini, G. S., et al. (2005). The ischemic rat heart releases S100B. Life Sciences, 77(8), 882-889.

25. Miyamoto, S., et al. (2008). Increased serum levels and expression of S100A8/A9 complex in infiltrated neutrophils in atherosclerotic plaque of unstable angina. Heart, 94(8), 1002-1007.

26. Most, P., \& Koch, W. J. (2007). S100A1: a calcium-modulating inotropic prototype for future clinical heart failure therapy. Future Cardiology, 3(1), 5-11.

27. Most, P., et al. (2004). Cardiac adenoviral S100A1 gene delivery rescues failing myocardium. The Journal of Clinical Investigation, 114(11), 1550-1563.

28. Most, P., et al. (2007). S100A1: a novel inotropic regulator of cardiac performance. Transition from molecular physiology to pathophysiological relevance. American Journal of Physiology. Regulatory, Integrative and Comparative Physiology, 293(2), R568-R577.

29. Pleger, S. T., et al. (2007). Targeting myocardial betaadrenergic receptor signaling and calcium cycling for heart 
failure gene therapy. Journal of Cardiac Failure, 13(5), 401414.

30. Pleger, S. T., et al. (2008). Endothelial S100A1 modulates vascular function via nitric oxide. Circulation Research, 102(7), 786-794.

31. Santamaria-Kisiel, L., Rintala-Dempsey, A. C., \& Shaw, G. S. (2006). Calcium-dependent and -independent interactions of the S100 protein family. The Biochemical Journal, 396(2), 201-214.

32. Schneider, M., et al. (2007). S100A4 is upregulated in injured myocardium and promotes growth and survival of cardiac myocytes. Cardiovascular Research, 75(1), 40-50.

33. Tsoporis, J. N., et al. (2005). S100B expression modulates left ventricular remodeling after myocardial infarction in mice. Circulation, 111(5), 598-606.

34. Tsoporis, J. N., et al. (2005). S100A6 is a negative regulator of the induction of cardiac genes by trophic stimuli in cultured rat myocytes. Experimental Cell Research, 303(2), 471-481.

35. Tsoporis, J. N., et al. (2003). Regulation of the S100B gene by alpha 1-adrenergic stimulation in cardiac myocytes. American Journal of Physiology. Heart and Circulatory Physiology, 284(1), $\mathrm{H} 193-\mathrm{H} 203$.

36. Usui, A., et al. (1990). S-100ao protein in serum during acute myocardial infarction. Clinical Chemistry, 36(4), 639-641.

37. Viemann, D., et al. (2005). Myeloid-related proteins 8 and 14 induce a specific inflammatory response in human microvascular endothelial cells. Blood, 105(7), 2955-2962.

38. Rustandi, R. R., et al. (2002). Three-dimensional solution structure of the calcium-signaling protein apo-S100A1 as determined by NMR. Biochemistry, 41(3), 788-796.

39. Osterloh, D., Ivanenkov, V. V., \& Gerke, V. (1998). Hydrophobic residues in the C-terminal region of S100A1 are essential for target protein binding but not for dimerization. Cell Calcium, 24 (2), 137-151.

40. Hare, J. M., \& Stamler, J. S. (2005). NO/redox disequilibrium in the failing heart and cardiovascular system. The Journal of Clinical Investigation, 115(3), 509-517.

41. Ehlermann, P., et al. (2000). Right ventricular upregulation of the $\mathrm{Ca}(2+)$ binding protein S100A1 in chronic pulmonary hypertension. Biochimica et Biophysica Acta, 1500(2), 249-255.

42. Haimoto, H., \& Kato, K. (1988). S100a0 (alpha alpha) protein in cardiac muscle. Isolation from human cardiac muscle and ultrastructural localization. European Journal of Biochemistry, 171(1-2), 409-415.

43. Kato, K., et al. (1986). S100a0 (alpha alpha) protein: distribution in muscle tissues of various animals and purification from human pectoral muscle. Journal of Neurochemistry, 46(5), $1555-1560$

44. Remppis, A., et al. (1996). Altered expression of the $\mathrm{Ca}(2+)-$ binding protein S100A1 in human cardiomyopathy. Biochimica et Biophysica Acta, 1313(3), 253-257.

45. Zimmer, D. B., Song, W., \& Zimmer, W. E. (1991). Isolation of a rat S100 alpha cDNA and distribution of its mRNA in rat tissues. Brain Research Bulletin, 27(2), 157-162.

46. Kato, K., \& Kimura, S. (1985). S100ao (alpha alpha) protein is mainly located in the heart and striated muscles. Biochimica et Biophysica Acta, 842(2-3), 146-150.

47. Kiewitz, R., et al. (2000). Transcriptional regulation of S100A1 and expression during mouse heart development. Biochimica et Biophysica Acta, 1498(2-3), 207-219.

48. Most, P., et al. (2006). Cardiac S100A1 protein levels determine contractile performance and propensity toward heart failure after myocardial infarction. Circulation, 114(12), 1258-1268.

49. Desjardins, J. F., et al. (2009). Lack of S100A1 in mice confers a gender-dependent hypertensive phenotype and increased mortality after myocardial infarction. American Journal of Physiology. Heart and Circulatory Physiology, 296(5), H1457-H1465.
50. Desjardins, J. F., Teichert-Kuliszewska, K., \& Parker, T. (2010). S100A1: A pluripotent regulator of cardiac and vascular function. Canadian Journal of Cardiology, 26(Suppl A), 9A-12A.

51. Lefranc, F., et al. (2005). Co-expression/co-location of S100 proteins (S100B, S100A1 and S100A2) and protein kinase C (PKC-beta, -eta and -zeta) in a rat model of cerebral basilar artery vasospasm. Neuropathology and Applied Neurobiology, 31(6), 649-660.

52. Kettlewell, S., et al. (2005). S100A1 increases the gain of excitation-contraction coupling in isolated rabbit ventricular cardiomyocytes. Journal of Molecular and Cellular Cardiology, 39(6), 900-910.

53. Kiewitz, R., et al. (2003). Ca2+-dependent interaction of S100A1 with the sarcoplasmic reticulum Ca2+-ATPase2a and phospholamban in the human heart. Biochemical and Biophysical Research Communications, 306(2), 550-557.

54. Most, P., et al. (2001). S100A1: A regulator of myocardial contractility. Proceedings of the National Academy of Sciences of the United States of America, 98(24), 13889-13894.

55. Most, P., et al. (2005). Distinct subcellular location of the Ca2+binding protein $\mathrm{S} 100 \mathrm{~A} 1$ differentially modulates $\mathrm{Ca} 2+$-cycling in ventricular rat cardiomyocytes. Journal of Cell Science, $118(\mathrm{Pt} 2)$, 421-431.

56. Boerries, M., et al. (2007). Ca2+-dependent interaction of S100A1 with F1-ATPase leads to an increased ATP content in cardiomyocytes. Molecular and Cellular Biology, 27(12), 4365-4373.

57. Brezova, A., Heizmann, C. W., \& Uhrik, B. (2007). Immunocytochemical localization of S100A1 in mitochondria on cryosections of the rat heart. General Physiology and Biophysics, 26(2), $143-149$.

58. Gutierrez-Cruz, G., Van Heerden, A. H., \& Wang, K. (2001). Modular motif, structural folds and affinity profiles of the PEVK segment of human fetal skeletal muscle titin. The Journal of Biological Chemistry, 276(10), 7442-7449.

59. Heierhorst, J., et al. (1996). Ca2+/S100 regulation of giant protein kinases. Nature, 380(6575), 636-639.

60. Maco, B., et al. (2001). Ultrastructural distribution of the S100A1 $\mathrm{Ca} 2+-$ binding protein in the human heart. Physiological Research, 50(6), 567-574.

61. Yamasaki, R., et al. (2001). Titin-actin interaction in mouse myocardium: passive tension modulation and its regulation by calcium/S100A1. Biophysical Journal, 81(4), 2297-2313.

62. Most, P., et al. (2003). Transgenic overexpression of the $\mathrm{Ca} 2+-$ binding protein S100A1 in the heart leads to increased in vivo myocardial contractile performance. The Journal of Biological Chemistry, 278(36), 33809-33817.

63. Volkers, M., et al. (2007). S100A1 decreases calcium spark frequency and alters their spatial characteristics in permeabilized adult ventricular cardiomyocytes. Cell Calcium, 41(2), $135-143$.

64. Volkers, M., et al. (2010). S100A1: A regulator of striated muscle sarcoplasmic reticulum $\mathrm{Ca} 2+$ handling, sarcomeric, and mitochondrial function. Journal of Biomedicine and Biotechnology, 2010,178614

65. Treves, S., et al. (1997). Interaction of S100A1 with the Ca2+ release channel (ryanodine receptor) of skeletal muscle. Biochemistry, 36(38), 11496-11503.

66. Leyva, J. A., Bianchet, M. A., \& Amzel, L. M. (2003). Understanding ATP synthesis: Structure and mechanism of the F1-ATPase (review). Molecular Membrane Biology, 20(1), 27 33.

67. Balaban, R. S. (2002). Cardiac energy metabolism homeostasis: Role of cytosolic calcium. Journal of Molecular and Cellular Cardiology, 34(10), 1259-1271.

68. Fukushima, H., Chung, C. S., \& Granzier, H. (2010). Titinisoform dependence of titin-actin interaction and its regulation by 
S100A1/Ca2+ in skinned myocardium. Journal of Biomedicine and Biotechnology, 2010, 727239.

69. Remppis, A., et al. (2002). The small EF-hand Ca2+ binding protein $\mathrm{S} 100 \mathrm{~A} 1$ increases contractility and $\mathrm{Ca} 2+$ cycling in rat cardiac myocytes. Basic Research in Cardiology, 97(Suppl 1), I56-I62.

70. Pleger, S. T., et al. (2007). Stable myocardial-specific AAV6S100A1 gene therapy results in chronic functional heart failure rescue. Circulation, 115(19), 2506-2515.

71. Pleger, S. T., et al. (2005). S100A1 gene therapy preserves in vivo cardiac function after myocardial infarction. Molecular Therapy, 12(6), 1120-1129.

72. Bristow, M. R. (2000). Beta-adrenergic receptor blockade in chronic heart failure. Circulation, 101(5), 558-569.

73. Lohse, M. J., Engelhardt, S., \& Eschenhagen, T. (2003). What is the role of beta-adrenergic signaling in heart failure? Circulation Research, 93(10), 896-906.

74. Kraus, C., et al. (2009). S100A1 in cardiovascular health and disease: closing the gap between basic science and clinical therapy. Journal of Molecular and Cellular Cardiology, 47(4), 445-455.

75. Pleger, S. T., et al. (2006). S100A1 gene transfer in myocardium. European Journal of Medical Research, 11(10), 418-422.

76. Pleger, S. T., Shan, C., Kziencek, J., Mueller, O., Bekeredjian, R., Remppis, A., et al. (2008). Retroinfusion-facilitated inotropic AAV9-S100A1 gene therapy restores global cardiac function in a clinically relevant pig heart failure model. Circulation, 118, S_792.

77. Du, X. J., et al. (2002). Impaired cardiac contractility response to hemodynamic stress in S100A1-deficient mice. Molecular and Cellular Biology, 22(8), 2821-2829.

78. Fulle, S., et al. (1999). Rapid desensitization of PC12 cells stimulated with high concentrations of extracellular S100. Neuroscience, 89(3), 991-997.

79. Hernandez-Ochoa, E. O., et al. (2009). Augmentation of Cav1 channel current and action potential duration after uptake of S100A1 in sympathetic ganglion neurons. American Journal of Physiology. Cell Physiology, 297(4), C955-C970.
80. Huttunen, H. J., et al. (2000). Coregulation of neurite outgrowth and cell survival by amphoterin and S100 proteins through receptor for advanced glycation end products (RAGE) activation. The Journal of Biological Chemistry, 275(51), 40096-40105.

81. Mariggio, M. A., et al. (1994). The brain protein S-100ab induces apoptosis in PC12 cells. Neuroscience, 60(1), 29-35.

82. Most, P., et al. (2003). Extracellular S100A1 protein inhibits apoptosis in ventricular cardiomyocytes via activation of the extracellular signal-regulated protein kinase 1/2 (ERK1/2). The Journal of Biological Chemistry, 278(48), 48404-48412.

83. Kiewitz, R., et al. (2000). S100A1, a new marker for acute myocardial ischemia. Biochemical and Biophysical Research Communications, 274(3), 865-871.

84. Rohde, D., Boerries, M., Herzog, N., Qiu, G., Ehlermann, P., Nawroth, P. P., et al. (2008). Cardiomyocyte damage-released S100A1 protein evokes a proinflammatory phenotype in cardiac fibroblasts. Circulation, 118, S_386.

85. Voekers, M., Weidenhammer, $\bar{C}$., Herzog, N., Friedrich, O., Fink, R. H. A., Koch, W. J., et al. (2008). S100A1 prevents arrhythmogenic diastolic sarcoplasmic reticulum calcium leak in ventricular cardiomyocytes. Circulation, 118, S_527.

86. Remppis, A., et al. (2004). S100A1 gene transfer: A strategy to strengthen engineered cardiac grafts. The Journal of Gene Medicine, 6(4), 387-394.

87. Polyakov, A. A., et al. (1998). Interaction of isoforms of S100 protein with smooth muscle caldesmon. FEBS Letters, 422(2), 235-239.

88. Daub, B., et al. (2003). Expression of members of the S100 Ca2+binding protein family in guinea-pig smooth muscle. Cell Calcium, 33(1), 1-10.

89. Mandinova, A., et al. (1998). Distinct subcellular localization of calcium binding S100 proteins in human smooth muscle cells and their relocation in response to rises in intracellular calcium. Journal of Cell Science, 111(Pt 14), 2043-2054.

90. Wright, N. T., et al. (2009). S100A1: structure, function, and therapeutic potential. Current Chemical Biology, 3(2), 138-145. 